\section{Variability in withdrawal of life-sustaining therapies}

Baseline patient acuity variables are insufficient to explain the observed variability in the withdrawal of life-sustaining therapies in severe traumatic brain injury. Hospital- and physicianbased variability in end-of-life decision-making and prognostication are well known. ${ }^{1-3}$ Turgeon and colleagues attempt to draw similar conclusions in severe traumatic brain injury in Canadian centres. ${ }^{4}$ Prognostication based on the physician, rather than on patient factors, is a concern to intensive care unit (ICU) practitioners. However, a number of important methodologic concerns cloud - rather than illuminate - in Turgeon and colleagues' retrospective study. ${ }^{4}$ Mortality ranged from $10.8 \%$ to $44.2 \%$ across different centres, with most deaths associated with the withdrawal of life-sustaining therapies. The variation in mortality rates, after adjustments for admission covariates (sex, age, pupillary reactivity and Glasgow Coma Scale score), was attributed to differences in withdrawal rates because of hospital and/or physician practices rather than patient acuity. Because baseline covariates did not appear to explain the differences in adjusted mortality rates, we are left to assume that no other clinical variables during ICU admission would have an impact on decision-making. These are strong conclusions in the absence of information regarding the severity and temporal evolution of the brain injury after ICU admission.

Turgeon and colleagues ${ }^{4}$ provide no data on comparative and serial neuroimaging between patients, neurosurgical and neuroprotective interventions, such as ventricular drainage and decompressive craniectomy, and other prognostic tests (e.g., somatosensory evoked potentials). Most concerning is the lack of information about intracranial hypertension, its measurement, management and the response to medical and surgical interventions. The ability for ICU physicians to neuroprognosticate is not limited to admission variables. Without information about the evolution of brain injury, intracranial pressure and response to interventions, the data provided do not support conclusions about the observed effect of medical practices on mortality rates.

\section{Sam D. Shemie MD}

Montreal Children's Hospital, McGill University Health Centre

\section{Patricia S. Fontela MD MSc}

The Department of Epidemiology, Biostatistics and Occupational Health, McGill University, Montréal, Que.

\section{References}

1. Rocker G, Cook D, Sjokvist P, et al.; Level of Care Study Investigators. Canadian Critical Care Trials Group. Clinician predictions of intensive care unit mortality. Crit Care Med 2004;32:1149-54.

2. Cook D, Rocker G, Marshall J, et al.; Level of Care Study Investigators and the Canadian Critical Care Trials Group. Withdrawal of mechanical ventilation in anticipation of death in the intensive care unit. $N$ Engl J Med 2003;349:1123-32.

3. Sprung CL, Cohen SL, Sjokvist P, et al.; Ethicus Study Group. End-of-life practices in European intensive care units: the Ethicus Study. JAMA 2003;290:790-7

4. Turgeon AF, Lauzier F, Simard JF, et al.; Canadian Critical Care Trials Group. Mortality associated with withdrawal of life-sustaining therapy for patients with severe traumatic brain injury: a Canadian multicentre cohort study. CMAJ 2011;183: 1581-8.

CMAJ 2012. DOI:10.1503/cmaj.112-2020

\section{The authors respond}

We agree with Drs. Shemie and Fontela ${ }^{1}$ that not all potential confounding variables could be considered in our study. ${ }^{2}$ We did adjust for the strongest baseline clinical predictors of death in this population, ${ }^{3,4}$ but as we indicated in our interpretation, variables that could not be evaluated may have also contributed to the observed variation (e.g., regional differences in referral patterns, casemix, religious beliefs or personal preferences). We agree that the evolution of brain injury over time often affects recommendations by physicians to withdraw life-sustaining therapies. However, we also observed large inter-hospital variability in rates of death due to withdrawal of life-sustaining therapies during the first three days of care - a time frame that is arguably too early to form accurate predictions about neuroprognosis. The observed inter-hospital variation cannot likely be explained by systematic differences in the temporal evolution of the brain injury across centres as suggested, and we remain concerned that differences in practice patterns are also likely to be responsible.
Although our study conclusions may generate discomfort, we believe that variation in physicians' perceptions of neurologic prognosis for patients with severe traumatic brain injury likely contribute to the observed variability in rates of death following the withdrawal of life-sustaining therapies. We believe our study helps highlight the need for high-quality research to better inform neuroprognostication, so that we can help families decide when to continue - and when to stop - life-sustaining treatments for these patients.

\section{Alexis F. Turgeon MD MSc}

Assistant Professor, Laval University,

Laval, Que.

Francois Lauzier MD MSc,

Damon C. Scales MD PhD,

Karen E.A. Burns MD MSc,

Lynne Moore PhD, David Zygun MD MSc, Francis Bernard MD,

Maureen O. Meade MD MSc,

Dean A . Fergusson PhD

\section{References}

1. Shemie SD, Fontela PS. Variability in withdrawal of life-sustaining therapies [letter]. CMAJ 2012; 184:326.

2. Turgeon AF, Lauzier F, Simard JF, et al.; Canadian Critical Care Trials Group. Mortality associated with withdrawal of life-sustaining therapy for patients with severe traumatic brain injury: a Canadian multicentre cohort study. CMAJ 2011;183: 1581-8. Published online 2011 Aug 29.

3. Perel P, Arango M, Clayton T, et al.; MRC CRASH Trial Collaborators. Predicting outcome after traumatic brain injury: practical prognostic models based on large cohort of international patients. BMJ 2008;336:425-9.

4. Steyerberg EW, Mushkudiani N, Perel P, et al. Predicting outcome after traumatic brain injury: development and international validation of prognostic scores based on admission characteristics. PLoS Med 2008;5(8):e165; discussion e165.

CMAJ 2012. DOI:10.1503/cmaj.112-2021

\section{Enforcement of natural health product regulations}

As a long-time advocate for a consistent, risk-based approach to health product regulation, Consumer Health Products Canada applauds the basic thrust of the CMAJ editorial "No regulatory double standard for natural health products." Consumer health products used in the practice of self-care can make a positive difference in the lives of Canadians and contribute greatly to the costeffectiveness of the Canadian health care system. However, natural health products can do this consistently only if 